\title{
AN INTERPRETATION OF THE WC STARS
}

\author{
LINDSEY F. SMITH
}

NASA Goddard Space Flight Centre, Greenbelt, Md., U.S.A.*

I wish to draw your attention to a (quite unexpected) property of the WC binaries. I believe that it gives us a clue to one of the parameters that controls the subclasses of the WR stars.

\section{Observations}

Table I collects the information available regarding the separations of all WR binaries for which there are adequate observations. The data are taken from the same references

TABLE I

Separations of Wolf-Rayet Binaries

\begin{tabular}{|c|c|c|c|c|c|c|c|}
\hline HD & Name & Sp. Type & $\begin{array}{l}a_{\mathrm{w}} \sin i \\
\left(R_{\odot}\right)\end{array}$ & $\begin{array}{l}a_{0} \sin i \\
\left(R_{\odot}\right)\end{array}$ & $\sin i$ & Basis of $\sin i$ & $A / R_{\odot}$ \\
\hline 186943 & & WN4 + B & $\left\{\begin{array}{l}29.7 \\
44.3\end{array}\right.$ & 12.6 & 0.77 & Assume $M_{\mathrm{wR}} \approx 11 M_{\odot}$ & $64:$ \\
\hline 190918 & & $\mathbf{W N}+09 \mathrm{I}$ & 108 & 17.6 & 0.36 & Assume $M_{\mathrm{wR}} \approx 11 M_{\odot}$ & 350 \\
\hline MR 114 & CX Ceph & WN5 & 12.1 & & $>0.94$ & Eclipsing & $26^{\mathrm{a}}$ \\
\hline 193576 & V444 Cyg & WN5 + O6 & 25.6 & 10.0 & 0.98 & Eclipsing & 36 \\
\hline 211853 & & WN6 + O6I & 27.5 & 13.1 & 0.96 & Eclipsing & 53 \\
\hline 228766 & & WN7 + O6V & 50 & $10.6::$ & $\approx 1$ & Large mass function & 61 \\
\hline 214419 & CQ Ceph & WN7 & $\left\{\begin{array}{l}9.6 \\
5.0\end{array}\right.$ & & $\approx 1$ & Eclipsing & $20: a^{a}$ \\
\hline 193793 & & $\mathrm{WC7}+\mathrm{O} 5$ & Period 3 & years $(\mathrm{Co}$ & nti, priva & communication large!) & \\
\hline 152270 & & $\mathrm{WC7}+$ O5-8 & 28.2 & 7.6 & 0.52 & Assume $M_{\mathrm{wR}} \approx 11 M_{\odot}$ & 69 \\
\hline 68273 & $\gamma_{2}$ Vel & $\mathrm{WC} 8+09 \mathrm{I}$ & 234 & 65.7 & 1 & $\begin{array}{l}\text { Large minimum } \\
\text { masses }\end{array}$ & 300 \\
\hline 168206 & CV Ser & WC8 + B0: & 86.5 & 26.4 & 1 & $\begin{array}{l}\text { Was eclipsing in } \\
\text { the past }\end{array}$ & 113 \\
\hline
\end{tabular}

assuming $M_{\mathrm{wR}} / M_{\mathrm{OB}}<1$

used to compile Table VI of Smith (1968a) and Table I of Kuhi (this Symposium). In many cases the inclination of the system is unknown; I have estimated it by assuming a mass of $11 M_{\odot}$ for the WR star. (Since the quantity derived from the observations is $M_{\mathrm{w}} \sin ^{3} i$, the derived value of $\sin i$ is not sensitive to the assumed mass, and the values obtained for $\sin i$ should be reasonably accurate.) It is immediately obvious

* Present address: Max Planck Institut für Radioastronomie, 53 Bonn, Germany. 
that the separations of the binaries containing WN stars are all, with one exception, less than $65 R_{\odot}$, while those of binaries containing WC stars are all greater than $65 R_{\odot}$, often much greater. I think we may safely deduce that the wide separation is a universal property of WC binaries and must be a consequence of the evolution that creates WC stars.

The difference in separation characteristic of $\mathrm{WN}$ and $\mathrm{WC}$ binaries provides an explanation for the observation that spectra of WC stars are apparently unaffected by the presence of a companion (see, for example, the spectra of WC5 binaries in the Large Magellanic Cloud shown by Smith, 1968b); whereas the spectra of WN stars in binary systems appear to be significantly different from those of single stars with comparable degree of excitation (see Hiltner and Schild, 1966). The WC binaries are sufficiently widely separated that the WC atmosphere is unperturbed by the presence of the companion, while the companions of WN stars are closer and gravitational perturbation, reflection effects and limitation of the atmosphere's extent by the Roche lobe (see Limber, 1968) may each have an effect. This suggestion is confirmed by the observation that the spectrum of the WN4 star in the very wide binary, HD 190918, shows little sign of perturbation by the companion - in particular, the line widths are the same as in the spectrum of the 'single' star, HD 187282.

\section{Interpretation}

The binary WR stars have certainly all undergone mass exchange according to the original suggestion of Paczynski (1967). The separation of a binary changes during the mass exchange. The final separation is usually greater than the initial separation; however, its value depends on the initial separation, initial masses and also on the unknown amount of mass loss to the system as a whole (Paczyński and Ziolkowski, 1967). Thus, it is not obvious what values of the initial parameters will yield the observed final separations. However, let us consider the simplest possibility, that the large final separations of WC binaries result from large initial separations. (I suspect that mass loss is probably also vitally important but, in as much as its effect is not yet clear, I will not pursue that thought at this time.) Thus, I suggest that the initially closer binary systems produce WN stars, and the initially wider binary systems produce WC stars. That the separation should cause such a difference is not unreasonable, since the initial separation and mass ratio defines the stage in the evolution of the primary at which mass exchange commences. If the binary is wide, mass exchange will commence later. In the most extreme case (case C - Weigert, 1968) mass exchange does not begin until after ignition of helium burning in the core of the star; such a situation has the attractive consequence of creating plenty of carbon and oxygen for subsequent production of the characteristic spectrum of the WC stars.

It is of interest to ask if the initial separation might also be important in the definition of the subclasses of the WN stars. If it is so, I suggest that the separation increases from left to right in Table VIII of my review paper. Adding the WC stars and the initial separation to that table produces Table II. 
LINDSEY F. SMITH

TABLE II

The WC stars

\begin{tabular}{|c|c|c|c|c|c|c|c|c|}
\hline $\begin{array}{l}M / M_{\odot} \\
17 \\
14 \\
11.5 \\
8.5 \\
7\end{array}$ & $\begin{array}{c}M_{\text {bol }} \\
-9.5 \\
-9.0 \\
-8.5 \\
-8.0 \\
-7.5\end{array}$ & $\begin{array}{c}\top \\
\text { WN8 } \\
\perp\end{array}$ & 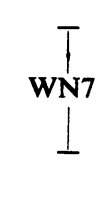 & $\underset{\perp}{\mathrm{WN}^{\top}}$ & $\begin{array}{c}{ }^{\top} \\
\text { WN6 } \\
\text { WN4 } \\
\perp\end{array}$ & $\begin{array}{c}\overline{1} \\
\text { WN5 } \\
\perp\end{array}$ & $\begin{array}{l}\text { WN5-C6 } \\
\text { WN6-C7 }\end{array}$ & $\begin{array}{c}\text { wC8 } \\
\text { Tं } \\
\text { wC6 } \\
\text { wC7 } \\
I\end{array}$ \\
\hline \multicolumn{2}{|c|}{$\begin{array}{c}(\mathrm{H} / \mathrm{He}) \\
T_{\text {eff }} \\
(\mathbf{K})\end{array}$} & $\begin{array}{c}\ll 2.3 \\
23000^{\circ}\end{array}$ & $<1.0$ & 0.8 : & $\left\{\begin{array}{r}0.4 \\
46000 \\
40000 \\
29000\end{array}\right.$ & $\begin{array}{r}0.0 \\
53000\end{array}$ & & $\begin{array}{r}0 ? \\
50000 ?\end{array}$ \\
\hline
\end{tabular}

Increasing Initial Separation

\section{Discussion}

I offer the following observations in support of the hypothesis that the order of the subclasses given in Table II represents a smooth progression of the basic stellar parameters and may be due to a progression of increasing initial separation.

(I) The intermediate $W N-W C$ types always have WN subclasses found at the right of the diagram, e.g. HD 62910, WN6-C7; HD 90657, WN4-C; HD 117688, WN6-C; MR 76, WC7-N6; NGC 6751, WC6-N5.

(II) The WN7 binary, $C Q C e p$ is the closest binary known, consistent with the position of the WN7 stars on the left side of the table. If a binary is initially very close, mass exchange can commence before exhaustion of hydrogen in the core of the star (Kippenhahn and Weigert, 1967; Case A); the comparatively high $\mathrm{H} / \mathrm{He}$ ratio in WN7 and WN8 stars may result from such an evolution. (It should be noted that the WN5 binary, CX Cep, also appears to have a very small separation, indicating that the scheme is not yet perfect.)

(III) The 'single' WN5 and WN6 stars commonly found in ring nebulae may be interpreted as very widely separated or disrupted binaries (see Paczynski, 1967), consistent with their place on the right side of the diagram. Note that, for disruption of a binary to occur, significant mass loss from the system as a whole is probably required. This may account for the presence of the nebulae around these particular subclasses, and also for the efficient removal of nearly all of the hydrogen from the surface of the star.

(IV) Hydrogen abundance decreases to the right among the WN stars. Thus, the suggested placement of the WC stars implies that they also have no hydrogen in their atmospheres. Kuhi and I have not attempted a derivation of the $\mathrm{H} / \mathrm{He}$ ratio of the WC stars because of the severe blending of carbon lines with the Pickering series. However, Paczynski, in his reveiw (this conference) gives us a choice between cosmic abundance and zero hydrogen for $\mathrm{WC}$ atmospheres. If the $\mathrm{H} / \mathrm{He}$ ratio were $10 / 1$, as 
for the cosmic abundance, then among the later lines of the Pickering series (which presumably eventually become optically thin) the even- $n$ lines would have 10 times the strength of the odd- $n$ lines. I think that, despite blending with carbon, such a contrast would be immediately noticeable. Thus, I anticipate that a $\mathrm{H} / \mathrm{He}$ ratio of $10 / 1$ will be ruled out by the observations.

It is worth noting that the strength of the helium lines is much less in the WC spectra than in the WN spectra; this is in accord with Paczyński's prediction that the helium abundance will begin to be significantly depleted in the extreme case where all the outer envelope of the star is lost, and the products of helium burning have been somehow mixed to the surface.

From the spectrum of $\gamma_{2}$ Velorum, Castor and Nussbaumer (1971) derive a lower limit to the number ratio of carbon to electrons of $2.510^{-3}$. That number is the order of magnitude expected if all hydrogen is gone and the helium is fully ionised. However, since it does not include $\mathrm{C}$ IV ions, Castor and Nussbaumer point out it may be an underestimate by a large factor.

(V) Temperature increases to the right among the WN stars. Thus, Table II implies that all the WC stars have very high photospheric temperatures. This may seem surprising considering the quite low excitation of spectra such as that of the WC 9 star in $\gamma_{2}$ Velorum. However, consider the fact that that star has a minimum mass of $15 M_{\odot}$ (Ganesh and Bappu, 1968). If it is a pure helium star, its bolometric magnitude should be about -9.2 . Thus, an observed visual absolute magnitude of -4.8 implies a bolometric correction of -4.4 and a temperature of $50000 \mathrm{~K}$.

The planetary nebulae associate with WC8 and WC9 nuclei are usually of quite low excitation. Johnson's suggestion (this Symposium) that the atmosphere of the WR star itself may be capable of 'smothering' the UV radiation is of interest in this regard. If the stars are as hot as I suggest, they must be quite small; $\simeq 15 R_{\odot}$ in the case of $\gamma_{2}$ Velorum. The atmosphere of $\gamma_{2}$ Velorum is, however, about $100 R_{\odot}$ (Hanbury Brown et al., 1970). (Note a distance of $460 \mathrm{pc}$ is used to obtain this value; Hanbury Brown et al. use $350 \mathrm{pc}$.) The large size of the atmosphere relative to the core together with the profusion of broad lines in all regions of WC spectra may mean that line opacity is dominant at all wavelengths and the amount of radiation escaping from the photosphere may be negligible. I think this is probably an equivalent statement to Johnson's 'smothering'.

It is of interest that, while the planetary nebulae around WC8 and WC9 nuclei are of low excitation, NGC 6751, which has a WC6 nucleus, is of moderately high excitation; thus, a temperature sequence like that among the WN stars may be indicated.

\section{Conclusions}

The observations are in reasonable accord with the suggestion that Table II represents a smooth progression of physical properties of the WR subclasses.

Ultimately, we would like to be able to arrange the classes according to the 'causal' parameters. To this end it has been suggested that the initial separation may be one of 
the more important such parameters and that its relationships to the subclasses and other final properties of the stars may be approximately as indicated. However, it seems certain, from both observational and theoretical considerations that a one-parameter family, as suggested by Table II, is an over-simplification. The evolution of the stars is probably sensitive not only to the initial separation, but also to the initial masses, angular momenta and chemical composition of the stars. The relationship of these initial parameters to the final subclasses, temperature, $\mathrm{H} / \mathrm{He}$ ratio, or separation may not be simple, or even single valued. Thus, the absence of an obvious and simple relationship between the final properties and the inferred initial properties is not surprising.

It would be an interesting experiment to deduce the initial configurations from the observed final configurations. The unknown amount of mass loss from the system represents a major uncertainty; however, calculations ignoring that problem would be a first step.

\section{Acknowl ${ }^{\mathbf{e}}$ dgements}

The manuscript was prepared while the author was at the Institut d'Astrophysique, Liège, and was supported by an ESRO Fellowship.

\section{References}

Castor, J. I. and Nussbaumer, H.: 1971, Monthly Notices Roy. Astron. Soc. 155, 293.

Ganesh, K. S. and Bappu, M. K. V.: 1968, Kodaikanal Obs. Bull. 183, A77.

Hanbury Brown, R., Davies, J., Herbison-Evans, D., and Allen, L. R.: 1970, Monthly Notices Roy. Astron. Soc. 148, 103.

Hiltner, W. A. and Schild, R. E.: 1966, Astrophys. J. 143, 770.

Kippenhahn, R. and Weigert, A.: 1967, Z. Astrophys. 65, 251.

Paczyński, B. E.: 1967, Acta Astron. 17, 355.

Paczyński, B. E. and Ziolkowski, J.: 1967, Acta Astron. 17, 7.

Limber, N. E.: 1968, in K. B. Gebbie and R. N. Thomas (eds.), Wolf-Rayet Stars, U.S. Government Printing Office, Washington, D.C., p. 233.

Smith, L. F.: 1968a, in K. B. Gebbie and R. N. Thomas (eds.), Wolf-Rayet Stars, U.S. Government Printing Office, Washington, D.C., p. 44.

Smith, L. F.: 1968b, Monthly Notices Roy. Astron. Soc. 140, 409.

Weigert, A.: 1968, Mitt. Astron. Ges. 25, 19.

\section{DISCUSSION}

Underhill: There is one problem. In some of the WC's, I think HD 192103 is one of them, $H \beta$, with the blends and all, is very considerably stronger than $\lambda 4541$.

Smith: Ten times?

Underhill: What has the factor of 10 to do with anything? If all the things that are making that line are pretty optically thick, it doesn't matter if you add one hundred times; you do not increase the intensity that much.

Smith: Yes, that is correct, and $\mathrm{H} \beta$ is still thick, but by the time you get down to the thin lines...

Underhill: I am not talking about any optically thin lines, I am talking about $\mathrm{H} \beta$ and $\lambda 4541$, both of which, I believe, are probably rather optically thick.

Smith: Yes, but there are high lines which are eventually thin.

Underhill: Try to remember that there is a very definite intensity difference between those two 
lines. And you are saying there should be practically none. I cannot interpret that intensity difference without going to the theory, because I am pretty certain that it is not an optically thin situation.

Smith: I agree. From the thick lines you cannot say anything because differences of line strengths of a factor 2 are more likely to be due to blending with $\mathrm{C}$ than with $\mathrm{H}$. A definite answer is only possible when the lines are thin so that the full factor 10 will show up if $\mathrm{H}$ is present in its cosmic abundance.

Conti: I have a comment here. First of all, if one wants to put an element like hydrogen in there, then, you must see it. Also another interpretation one could have instead of the initial separation arguments which goes exactly the same way, is that the change in the period is a clue to the change in the mass ratio.

Smith: Yes, I am aware of that. That is the other possibility.

Conti: This argument says that a WC star would have peeled off more of its envelope, and become further separated.

Underhill: Were you first trying to infer that it is not true that the intensity of $\mathrm{H} \beta$ is not strong, considering the blends?

Conti: I was just saying you can play games if you see something or if you do not see something, even before you have a theory. But you cannot say "just because you do not see it, it is there, although I do not have a theory to explain it".

Underhill: You cannot say it is not there, if you consider that things are pretty well optically thick.

Conti: No, but you cannot say that it is there, if you do not see it.

Thomas: But do you believe that there is hydrogen in these stars?

Underhill: It depends on what 'seeing' is. What do you mean by 'seeing' something?

Conti: Hydrogen is seen in every star, except in these kinds of stars. That means there is definite observational evidence.

Thomas: Let me remind you the remark that Shapley once made, in discussing solar physics. He said, "Once you have seen one of Menzel's invisible prominences, you have seen them all". I think that is a very appropriate thing here.

Niemela: There are four stars, in Lindsey Smith's Catalogue, classified as intermediate objects. I took spectra of two of them, HD 117688 and HD 90657, and I could not confirm that they really are intermediate objects, they are just normal WN stars. HD 117688 was classified as an intermediate object, because of the emission line at $\lambda 4325$ which was assigned to $C$, but as you can see in Figure 1 , the line is present in other normal WN stars: it is actually a $\mathrm{N}$ III line, not a $\mathrm{C}$ line. Another reason for the classification as an intermediate object, was that the $\mathrm{N}$ III emission at $\lambda \lambda 4634-40-41$ had a contribution from $C$. On Figure 2 you can see that the line is centered at $\lambda 4638$, so it should be N m.

Smith: I can always be wrong. HD 117688 is one of the milder cases where the C contribution appeared to me much weaker than the N contribution. However, in cases such as HD 62910 and MR 76 I think there is little doubt, the spectra look like double exposures between WN and WC stars. I am very glad that you have continued the investigation, because it has obvious relevance to this somewhat revolutionary proposal I just made.

Niemela: Another thing I need to point out are those $V / R$ variations I was talking about in the first part of the discussion. They are seen in the Balmer lines, and in the He I lines, but not in He II. Could it mean that there is hydrogen in $\gamma_{2}$ Velorum?

Smith: Yes, that is the alternative explanation, of course. A naive interpretation of the mass exchange theory might suggest that if the separation is wider you may get more hydrogen left on the surface. My feeling was that the scheme presented fits the observations more consistently. Hopefully the theoreticians can solve this one, if the observations cannot.

Underhill: The answer to this question of leaving hydrogen on the surface, I think is pointed well towards the direction of hydrogen being on the surface, when you look at the spectra of two X-ray sources, that have optical spectra. There you see very strong hydrogen lines in emission as well as typical $\mathrm{C}, \mathrm{He} \mathrm{I}, \mathrm{O} \mathrm{V}$ and so on. There is no doubt about it: nobody has ever questioned that the hydrogen is there. Perhaps you would say, an X-ray source is a very old star, a helium star, very far long in evolution. However, it managed to keep some hydrogen which shows in the spectrum. That qualifies it, by the definition of the Wolf-Rayet spectrum, as a Wolf-Rayet object.

Conti: There is a theory I heard very recently about X-ray sources, which suggests that an X-ray source is a binary system in which material is falling in to one star, and, of course, that would all be hydrogen. So, the argument can go either way. 


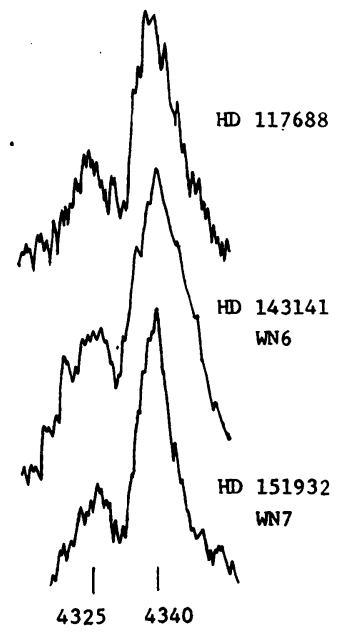

Fig. 1.

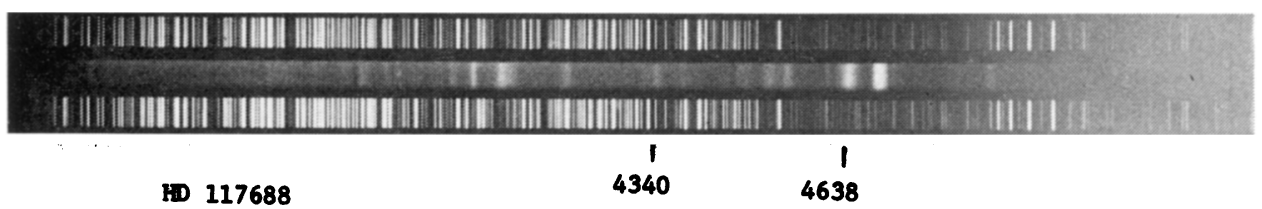

Fig. 2.

Van Blerkom: Can I ask about the temperature in the envelope of the X-ray source. It must be very hot.

Underhill: The region that is creating the X-rays, according to one calculation I read, is between $10^{7}$ and $10^{8}$ degrees and $N_{e}$ is $10^{16}$. The spectrum that you see, the Wolf-Rayet like spectrum looks like a very cool WC, except that it has very strong hydrogen lines.

Van Blerkom: The fact that you see hydrogen strongly in emission means that it probably has not been ionized away, even in these very hot envelopes, and it certainly would not be ionized away in the cooler Wolf-Rayet star.

Underhill: It would be an interesting question; exactly what happens to it and how it emits so strongly. So, we have now a question. For every Wolf-Rayet star, and there are some where there is very definitely $\mathrm{H} \beta$ stronger than $\lambda 4541$, can we always throw in a third star to provide us with the hydrogen? 